\title{
Staphylococcus lugdunensis: Review of Epidemiology, Complications, and Treatment
}

\author{
Shridhar Parthasarathy ${ }^{1}$, Shrey Shah ${ }^{1}$, Avinaash Raja Sager ${ }^{2}$, Anvitha Rangan ${ }^{2}$, Satya Durugu ${ }^{3}$
}

1. Biology, The College of New Jersey, Ewing, USA 2. Internal Medicine, Jawaharlal Nehru Medical College, Belgaum, IND 3. Internal Medicine, University of Louisville, Louisville, USA

Corresponding author: Shridhar Parthasarathy, shridparth@gmail.com

\begin{abstract}
Staphylococcus lugdunensis is a species of coagulase-negative staphylococci (CNS) that induces a variety of infectious diseases, including skin and soft tissue infection (SSTI), infective endocarditis (IE), and bone and PII. This review article underscores the important points in the literature about $S$. lugdunensis infections, including its epidemiology, diagnosis, and treatment, as well as specific types of infections it can cause. Anatomical and age-related distributions of $S$. lugdunensis SSTIs have been noted, though they most commonly occur as abscesses. S. lugdunensis can also manifest as an aggressive form of IE presenting with valve destruction and abscess formation, frequently requiring surgery and with a high mortality rate. Bone and joint infections caused by $S$. lugdunensis are also more invasive than infections by other species of CNS. The clinical presentation of $S$. lugdunensis infection in SSTI, IE, and bone/joint infection is frequently more similar to that of S. aureus infection than that of other CNS infections, necessitating species-level differentiation of CNS for proper diagnosis. Though historically, this depended upon biochemical tests that were neither routine nor reliable, the implementation of matrix-assisted laser desorption/ionization time of flight mass spectrometry (MALDI-TOF MS) in clinical laboratories has made identification of CNS species such as $S$. lugdunensis more practical. Imaging modalities, especially the fluorodeoxyglucose (FDG) with positron emission tomography (PET), are another important emerging trend in the diagnosis of infectious diseases such as $S$. lugdunensis infection. S. lugdunensis remains highly susceptible to a wide gamut of antibacterial therapies, which is uncharacteristic of other CNS. Infections can usually be treated by antibiotics traditionally used for CNS such as oxacillin. The breakpoints for S. lugdunensis are higher than those of other CNS and similar to $S$. aureus breakpoints. In the case of aggressive IE or bone/joint infection by $S$. lugdunensis, it is recommended to treat with a $\beta$-lactam agent. Further study is needed to understand the diversity, virulence, and population structure of this species, as well as its role in other infections, such as urinary tract infections (UTIs), respiratory infections, peritonitis, and bacteremia.
\end{abstract}

Received 06/08/2020

Review began 06/18/2020 Review ended 06/24/2020 Published 06/24/2020

(c) Copyright 2020 Parthasarathy et al. This is an open access article distributed under the terms of the Creative Commons Attribution License CC-BY 4.0., which permits unrestricted use, distribution, and reproduction in any medium, provided the original author and source are credited.
Categories: Internal Medicine, Infectious Disease, Healthcare Technology

Keywords: staphylococcus lugdunensis, coagulase-negative staphylococci, infective endocarditis, bone infection, prosthetic joint infection, skin and soft tissue infection, pet

\section{Introduction And Background}

Staphylococcus lugdunensis is a species of coagulase-negative staphylococci (CNS), known to be a normal skin commensal that preferentially colonizes the perineal region [1-3]. In recent years, a growing body of evidence demonstrates the role of $S$. lugdunensis in a wide spectrum of diseases. Though the most common of these are skin and soft tissue infections (SSTIs), S. lugdunensis has also been shown to infrequently cause bone, joint space, and prosthetic joint infections (PIIs); aggressive valvular endocarditis with significant mortality rates; urinary tract infections; and peritonitis [1,4-15]. These infections may differ greatly from other CNS infections in clinical presentation and treatment options; hence, an understanding of specific features of $S$. lugdunensis infection has particular clinical value.

This review article aims to highlight salient points pertaining to S. lugdunensis within the existing literature, particularly relating to its pathogenesis, microbiology, treatment modalities, and emerging trends.

\section{Review}

\section{Epidemiology}

S. lugdunensis physiological colonization is estimated to be present in $30 \%$ to $50 \%$ of patients, mostly in the inguinal area with a substantial presence in the axilla and nares as well [16]. It has a low presence in human clinical samples. A review of studies between 2001 and 2011 in human clinical samples found S. lugdunensis presence ranging from $0.5 \%$ to $9 \%$ in CNS-positive samples, while one study in 2015 also identified $3.6 \%$ of CNS-positive blood cultures to be S. lugdunensis [17,18]. Other studies have consistently shown S. lugdunensis to represent under $3 \%$ of CNS in human samples [19]. The incidence of S. lugdunensis SSTIs is estimated to be 53 per 100,000 per year [1]. S. lugdunensis bacteremia (SLB) incidence has been estimated at 5.6 patients per 100,000 admissions, while the incidence of clinically relevant SLB was 1.3 patients per 100,000 admissions [20]. 
S. lugdunensis is nonetheless an important pathogen. It has been reported as the second most common pathogen, behind S. epidermidis, in infective endocarditis (IE) resulting from CNS [21]. In a study in which other CNS species were microbiologically relevant in under $26 \%$ of cases, S. lugdunensis had $40 \%$ microbiological relevance [22]. In another, seven of eight identified cases of SLB were determined to be clinically significant $[18]$.

\section{Skin and soft tissue infection}

SSTIs account for the majority of $S$. lugdunensis infections, with Bocher et al., as described above, having identified an incidence of 53 per 100,000 per year, representing an increase from earlier pilot studies $[1,4,23]$. Though less common than SSTIs caused by other CNS and S. aureus, it produces a more virulent clinical picture in contrast to the CNS and more closely resembling that of $S$. aureus [1].

These infections commonly affect the middle-aged to elderly patient populations, with greater prevalence in females $[1,5]$. Approximately half of all patients affected have some form of concurrent comorbidity, either in the form of chronic immunosuppressive therapy, diabetes mellitus, or a history of trauma to the site $[4,5]$. Anatomically, previous studies have shown S. lugdunensis infections to be distributed predominantly below the pelvic girdle or in the inguinal area, though Herchline and Ayers noted a broader spectrum of localization $[4,24,25]$. This is supported and built on by Bocher et al., who posited an age-related distribution of SSTI sites: among children, otitis externa is most common; among the middle-aged, apocrine glandrelated areas such as the axilla, buttocks, groin and mammary regions; and among the elderly, infections and ulcers of the digits [1].

Abscesses are the most common manifestation of $S$. lugdunensis infections [1]. In a case series of five patients with cutaneous $S$. lugdunensis infection, Heldt Manica and Cohen also noted cellulitis, cyst formation, or pustules as a mode of presentation. All five patients presented without any other constitutional symptoms. Almost all patients require incision and drainage along with antibiotic therapy; and the rare few requiring only monotherapy are those with superficial infections [1,5]. S. lugdunensis demonstrated an antibiotic sensitivity similar to methicillin-sensitive $S$. aureus, and some patients may require prolonged therapy [5].

\section{Infective endocarditis}

Infrequently, $S$. lugdunensis manifests as an aggressive form of IE presenting with valve destruction and abscess formation. In a literature review ranging from 1988 to 2008 with a total of 67 documented cases, Liu et al. found that $S$. lugdunensis IE predominantly affected the left side of the heart and formed vegetations demonstrable on echocardiography [9]. These infections were more prevalent in males, and the majority of infected patients were middle-aged with a history of comorbidity [9].

In contrast to other CNS infections, which tend to be acquired at the hospital and to affect prosthetic valves and indwelling devices, $S$. lugdunensis mainly affects native heart valves and is more likely to be acquired through the community without an identifiable source of infection $[9,26]$. The presentation of S. lugdunensis IE has been noted to more closely resemble that of $S$. aureus IE compared to IE due to other CNS species [9].

Antibiotic therapy alone is usually not sufficient and patients often require surgical interventions with valve replacement. The need for surgery is much higher than that of $S$. aureus IE (70\% vs. 37\%) and comparable to that of $S$. epidermidis IE - though with significantly higher mortality rates [9,27]. Surgery is the only independent risk factor for mortality [9]. Unlike other CNS, most cases of S. lugdunensis are susceptible to a wide array of antimicrobials especially penicillins, though conservative management alone is often never satisfactory [9]. The Infectious Diseases Society of America guidelines for endocarditis due to S. lugdunensis recommends $\beta$-lactam agent treatment along with monitoring for the development of either periannular extension or extracardiac spread of infection [28].

\section{Native bone, prosthetic joint and vertebral space infections}

Another rising trend is the involvement of S. lugdunensis in orthopedic diseases. S. lugdunensis mostly affects prosthetic joints causing PJIs, and an equal amount of native joint and spondylodiscitis/vertebral osteomyelitis. These infections are more prevalent in males, and typically manifest in middle age [7]. In PJIs, the knee joint is more frequently affected than the hip joint [6].

In a study of the largest cohort of its kind, Shah et al. found that PJIs due to S. lugdunensis tend to be more invasive than other CNS species and are more similar to those due to $S$. aureus: infections presented with an acute clinical picture of copious purulent discharge and rapid tissue destruction [6]. A majority of patients have underlying comorbidities or a history of immunosuppression, including those due to diabetes mellitus, chronic steroid therapy, or, most commonly, some form of urogenital anomaly [6,7]. This relation to underlying urogenital anomalies is well documented in other literature as well, though its role cannot be established without case-control studies [3,4,28,29]. The majority of patients with bone/joint space infections and PJI require surgical intervention along with parenteral antibiotic therapy [6,7]. A noteworthy exception is in the case of spondylodiscitis and vertebral osteomyelitis, where successful resolution may be achieved without surgery [7]. 
Based on current Clinical Laboratory Standards Institute guidelines, an S. aureus interpretative breakpoint for oxacillin should be used, meaning a MIC of $1 \mathrm{microgram} / \mathrm{ml}$ is deemed oxacillin sensitive for $S$. lugdunensis but resistant for other CNS $[6,23]$. $\beta$-lactams are preferred over vancomycin due to their more rapid bactericidal action and better penetration into bone, and display fewer adverse effects, such as hypotension and red man syndrome, compared to vancomycin [30-32]. Patients treated with parenteral $\beta$ lactams are also more likely to be free of treatment failure at a two-year interval than those who received parenteral vancomycin [6].

\section{Diagnosis}

Diagnosing a CNS such as $S$. lugdunensis begins with a clinical presentation suspicious for a staphylococcus infection, which is frequently expected to be a coagulase-positive staphylococcus infection (e.g., S. aureus). A bacterial culture is then performed in which CNS can be detected; however, CNS detection alone may not be sufficient to determine clinical significance. This difficulty arises from the possibility that a CNS-positive culture may result from contamination of the specimen or colonization of skin or mucous membranes rather than a clinically relevant infection [17]. For example, S. lugdunensis is also commonly isolated as a part of a mixed flora [14]. Often, a second positive culture result is also obtained before a CNS infection is diagnosed [33].

Historically, identifying individual CNS species has not been common in clinical laboratories. Distinguishing CNS from $S$. aureus, however, has been traditionally achieved by assays based on staphylococcal coagulase, or clumping factor. In particular, the clumping factor has been detected by the slide coagulase test or latex agglutination test, both of which detect both free and membrane-bound forms of coagulase. S. lugdunensis, which lacks free coagulase but, in up to $65 \%$ of isolates, possesses a membrane-bound form, is often mistaken for $S$. aureus following such tests as a result [34]. The tube coagulation test, which is more specific to free coagulase, is a traditional and more reliable method for CNS detection, but it is limited by long incubation time. The "third-generation," rapid latex, and hemagglutination assays that have been developed to circumvent these constraints are, however, themselves limited by lower specificity for $S$. aureus identification, likely due to clumping factor-positive CNS species including S. lugdunensis [17].

Species-level identification of $S$. lugdunensis depends on its specific diagnostic characteristics. This species forms cream-white to slightly yellow colonies is oxidase-negative and is not novobiocin-resistant [17]. Cultures commonly produce a 'hypochlorite bleach odor' similar to that of Eikenella corrodens in Colombia agar [1]. Additionally, Kleiner et al. and Liu et al. have noted the ability of S. lugdunensis to form biofilm and a glycocalyx coat, respectively, lending to its virulence [9,23,35]. Additionally, S. lugdunensis is positive for ornithine decarboxylase activity and pyrrolidonyl arylamidase activity in over $90 \%$ of isolates, and this test can yield results within eight hours [19]. Additionally, nucleic acid-based assays, including real-time polymerase chain reaction (PCR) for specific conserved genes such as the $16 \mathrm{~S}$ or 23S ribosomal DNA, have a higher identification rate for S. lugdunensis [16]. These tests have not been established as routine procedures, but are especially useful in the event of inconclusive or unclear results from other procedures [17].

Over the past decade, the increasing implementation of matrix-assisted laser desorption/ionization time of flight mass spectrometry (MALDI-TOF MS) in laboratories has resulted in simpler, faster, more costeffective, and increasingly accurate $S$. lugdunensis identification. This proteomic method relies on manufacturers' databases whose spectra content is growing, resulting in nearly $100 \%$ sensitivity and specificity [16]. Furthermore, MALDI-TOF MS can reliably identify S. lugdunensis directly from blood cultures [16]. The benefits of this test include fewer preparatory steps, as well as significantly reduced time since identification can be achieved within one hour [36]. Implementation of MALDI-TOF MS has resulted in drastic increases in S. lugdunensis detection in multiple studies. In one case, routine use of this technique coincided with a notable uptick in diagnosed $S$. lugdunensis urinary tract infections [37]. In another, there was an 18-fold increase in $S$. lugdunensis identification in the two years following MALDI-TOF MS implementation compared to the preceding two years [22]. Evidently, MALDI-TOF MS has established itself as a prime tool for identifying CNS at the species level and is therefore invaluable for the diagnosis of $S$. lugdunensis infection.

In recent years, imaging modalities have also shown to be effective in diagnosing infections, namely through the combined use of fluorodeoxyglucose (FDG) with positron emission tomography (PET) [38,39]. There has been an increasing trend to utilize FDG-PET in non-oncological fields such as infectious disease, cardiology, neurology, and gastroenterology [40-43]. FDG is a tracer which, like glucose, is uptaken in greater quantities by cells displaying higher metabolic activity. Coupling FDG with PET imaging, which has greater spatial resolution than alternative techniques, yields valuable molecular-level information for a variety of disorders [38]. Specifically, by localizing in regions of increased metabolic activity, FDG can delineate abnormalities in the body, including inflammatory response to infectious diseases, and is therefore an important diagnostic tool for conditions such as $S$. lugdunensis infection.

\section{Treatment}

In the treatment of CNS such as S. lugdunensis, the first line of antibacterial treatment is isoxazolyl penicillin, such as oxacillin. Fortunately, $S$. lugdunensis remains highly susceptible to a wide gamut of 
antibacterial therapies, which is uncharacteristic of other CNS such as $S$. epidermidis [34]. However, previous literature indicates the development of resistance to streptomycin, erythromycin, ceftazidime, and gentamicin through isolated case reports [16,34]. Also, Kragsbjerg et al. describe in a case report the development of resistance in $S$. lugdunensis to rifampicin and ciprofloxacin while treating a chronic $S$. lugdunensis infection [44]. However, successful treatment of $S$. lugdunensis with linezolid after the failure of ciprofloxacin and rifampin have been reported in a patient with a PJI [45]. Furthermore, S. lugdunensis resistance to penicillin has reported being considerably high at 45\% in the United States [16]. The Clinical Laboratory and Standards Institute (CLSI) has recommended that S. lugdunensis isolates should be screened for the penicillin-binding protein $2 \mathrm{~A}$ by latex agglutination or the mecA gene by PCR, both of which confer resistance to oxacillin and other $\beta$-lactams [46]. Additionally, CLSI guidelines also state breakpoints for $S$. lugdunensis that are higher than those of most other CNS [46]. Additionally, S. lugdunensis and S. aureus have the same breakpoints: susceptible isolates have MICs of $\leqslant 2 \mu \mathrm{g} / \mathrm{ml}$, while resistant isolates have MICs $\geqslant 4$ $\mu \mathrm{g} / \mathrm{ml}$. Vancomycin resistance in S. lugdunensis has not been documented to this date [46].

SSTIs of $S$. lugdunensis are indistinguishable visually from $S$. aureus. However, compared to other CNS, $S$. lugdunensis is associated with a higher complication rate [1]. Anguera et al. observed in a prospective cohort of ten patients with IE caused by $S$. lugdunensis that there was no difference in mortality between antibacterial monotherapy and antibacterial combination therapy [47]. However, there exists a lack of data in the literature on the efficacy of antibacterial treatments for S. lugdunensis infections currently. In terms of treatment, a vast majority of patients with a $S$. lugdunensis infection were treated primarily with surgical incision or antibiotics, while most other patients had superficial wound infections [1].

S. lugdunensis, like other CNS, is able to produce a biofilm that makes infections become significantly more difficult to treat even with the high susceptibility of S. lugdunensis to most antibiotic treatments [1]. Unfortunately, little is known about the diversity, virulence, and population structure of $S$. lugdunensis. Even though $S$. lugdunensis is highly susceptible to a myriad of antibiotic therapies, S. lugdunensis is more aggressive than other CNS as $S$. lugdunensis carries a high mortality rate in endocarditis patients [16]. Thus, if $S$. lugdunensis is isolated, transesophageal echocardiography should be conducted to evaluate for IE [4850]. Treatment with a $\beta$-lactam and debridement of foreign material should be pursued [48-50].

\section{Conclusions}

S. lugdunensis is not an uncommon cause of infection. Its occurrence warrants our further attention due to its significant virulence and a broad range of pathology. Previous literature highlights some of the important aspects of this organism - particularly its predilection for SSTIs, aggressive clinical course, a possible relationship with immunosuppression/comorbidity, and a wide spectrum of antibiotic susceptibilities. The significant differences in presentation and management of infections by $S$. lugdunensis compared to other staphylococcal infections necessitate species-level differentiation of these organisms, a process which has recently become much more efficient, reliable, and routine due to the implementation of MALDI-TOF MS in clinical laboratories. Furthermore, though possessing a wide array of antibiotic susceptibilities due to low genetic diversity, S. lugdunensis has an evolving resistance that must be highlighted.

Future studies should search and identify biases and shortcomings of current literature on S. lugdunensis and improve on them, in the hope of consolidating the clinical correlations mentioned in this article. Lastly, more studies are required to shed some light on the role of this unique organism in causing UTIs, respiratory infections, peritonitis, and bacteremia.

\section{Additional Information \\ Disclosures}

Conflicts of interest: In compliance with the ICMJE uniform disclosure form, all authors declare the following: Payment/services info: All authors have declared that no financial support was received from any organization for the submitted work. Financial relationships: All authors have declared that they have no financial relationships at present or within the previous three years with any organizations that might have an interest in the submitted work. Other relationships: All authors have declared that there are no other relationships or activities that could appear to have influenced the submitted work.

\section{References}

1. Bocher S, Tonning B, Skov RL, Prag J: Staphylococcus lugdunensis, a common cause of skin and soft tissue infections in the community. J Clin Microbiol. 2009, 47:946-950. 10.1128/JCM.01024-08

2. Bellamy R, Barkham T: Staphylococcus lugdunensis infection sites: predominance of abscesses in the pelvic girdle region. Clin Infect Dis. 2002, 35:e32-e34. 10.1086/341304

3. Van der Mee-Marquet N, Achard A, Mereghetti L, Danton A, Minier M, Quentin R: Staphylococcus lugdunensis infections: high frequency of inguinal area carriage. J Clin Microbiol. 2003, 41:1404-1409. 10.1128/jcm.41.4.1404-1409.2003

4. Herchline TE, Ayers LW: Occurrence of Staphylococcus lugdunensis consecutive clinical cultures and relationship of isolation to infection. J Clin Microbiol. 1991, 29:419-421.

5. Heldt Manica LA, Cohen PR: Staphylococcus lugdunensis infections of the skin and soft tissue: a case series 
and review. Dermatol Ther. 2017, 7:555-562. 10.1007/s13555-017-0202-5

6. Shah NB, Osmon DR, Fadel H, et al.: Laboratory and clinical characteristics of Staphylococcus lugdunensis prosthetic joint infections. J Clin Microbiol. 2010, 48:1600-1603. 10.1128/JCM.01769-09

7. Karnani R, Myers JP: Bone and joint infections caused by Staphylococcus lugdunensis: report of 2 cases and review of the literature. Infect Dis Clin Pract. 2008, 16:94-99. 10.1097/IPC.0b013e31816379ea

8. Douiri N, Hansmann Y, Lefebvre N, et al.: Staphylococcus lugdunensis: a virulent pathogen causing bone and joint infections. Clin Microbiol Infect. 2016, 22:747-748. 10.1016/j.cmi.2016.05.031

9. Liu PY, Huang YF, Tang CW, et al.: Staphylococcus lugdunensis infective endocarditis: a literature review and analysis of risk factors. J Microbiol Immunol Infect. 2009, 43:478-484. 10.1016/S1684-1182(10)60074-6

10. Jones RM, Jackson MA, Ong C, Lofland GK: Endocarditis caused by Staphylococcus lugdunensis . Pediatr Infect Dis J. 2002, 21:265-268. 10.1097/00006454-200203000-00025

11. Patel R, Piper KE, Rouse MS, Uhl JR, Cockerill FR 3rd, Steckelberg JM: Frequency of isolation of Staphylococcus lugdunensis among staphylococcal isolates causing endocarditis: a 20-year experience. J Clin Microbiol. 2000, 38:4262-4263.

12. Schonheyder HC, Hansen VK, Asschenfeldt P, Rosdahl VT: Staphylococcus lugdunensis: an important cause of endocarditis: a case report. APMIS. 1993, 101:802-804. 10.1111/j.1699-0463.1993.tb00183.x

13. Vandenesch F, Etienne J, Reverdy ME, Eykyn SJ: Endocarditis due to Staphylococcus lugdunensis: report of 11 cases and review. Clin Infect Dis. 1993, 17:871-876. 10.1093/clinids/17.5.871

14. Haile DT, Hughes J, Vetter E, et al.: Frequency of isolation of Staphylococcus lugdunensis in consecutive urine cultures and relationship to urinary tract infection. J Clin Microbiol. 2002, 40:654-656. 10.1128/JCM.40.2.654-656.2002

15. Schnitzler N, Meilicke R, Conrads G, Frank D, Haase G: Staphylococcus lugdunensis: report of a case of peritonitis and an easy to perform screening strategy. J Clin Microbiol. 1998, 36:812-813. 10.1128/JCM.36.3.812-813.1998

16. Argemi X, Hansmann Y, Riegel P, Prévost G: Is Staphylococcus lugdunensis significant in clinical samples? . J Clin Microbiol. 2017, 55:3167-3174. 10.1128/JCM.00846-17

17. Becker K, Heilmann C, Peters G: Coagulase-negative staphylococci. Clin Microbiol Rev. 2014, 27:870-926. 10.1128/CMR.00109-13

18. Sato M, Kubota N, Horiuchi A, Kasai M, Minami K, Matsui H: Frequency, clinical manifestations, and outcomes of Staphylococcus lugdunensis bacteremia in children. J Infect Chemother. 2016, 22:298-302. 10.1016/j.jiac.2016.01.012

19. Frank KL, Del Pozo JL, Patel R: From clinical microbiology to infection pathogenesis: how daring to be different works for Staphylococcus lugdunensis. Clin Microbiol Rev. 2008, 21:111-133. 10.1128/CMR.0003607

20. Choi SH, Chung JW, Lee EJ, et al.: Incidence, characteristics, and outcomes of Staphylococcus lugdunensis bacteremia. J Clin Microbiol. 2010, 48:3346-3349. 10.1128/JCM.00609-10

21. Petti CA, Simmon KE, Miro JM, et al.: Genotypic diversity of coagulase-negative staphylococci causing endocarditis: a global perspective. J Clin Microbiol. 2008, 46:1780-1784. 10.1128/JCM.02405-07

22. Argemi X, Riegel P, Lavigne T, et al.: Implementation of matrix-assisted laser desorption ionization-time of flight mass spectrometry in routine clinical laboratories improves identification of coagulase-negative staphylococci and reveals the pathogenic role of Staphylococcus lugdunensis. J Clin Microbiol. 2015, 53:2030-2036. 10.1128/JCM.00177-15

23. Kleiner E, Monk AB, Archer GL, Forbes BA: Clinical significance of Staphylococcus lugdunensis isolated from routine cultures. Clin Infect Dis. 2010, 51:801-803. 10.1086/656280

24. Hellbacher C, Tornqvist E, Soderquist B: Staphylococcus lugdunensis: clinical spectrum, antibiotic susceptibility, and phenotypic and genotypic patterns of 39 isolates. Clin Microbiol Infect. 2006, 12:43-49. 10.1111/j.1469-0691.2005.01296.x

25. Vandenesch F, Eykyn SJ, Etienne J, Lemozy J: Skin and post-surgical wound infections due to Staphylococcus lugdunensis. Clin Microbiol Infect. 1995, 1:73-74. 10.1111/j.1469-0691.1995.tb00449.x

26. Huebner J, Goldmann DA: Coagulase-negative staphylococci: role as pathogens. Annu Rev Med. 1999, 50:223-236. 10.1146/annurev.med.50.1.223

27. Ling ML, Yeo M: Staphylococcus lugdunensis: report of first case of skin and soft tissue infection in Singapore. Singapore Med J. 2000, 41:177-178.

28. Baddour LM, Wilson WR, Bayer AS, et al.: Infective endocarditis in adults: diagnosis, antimicrobial therapy, and management of complications. Circulation. 2015, 132:1435-1486. 10.1161/CIR.0000000000000296

29. Shuttleworth R, Colby WD: Staphylococcus lugdunensis endocarditis. J Clin Microbiol. 1992, 30:1948-1952.

30. Bolon MK, Morlote M, Weber SG, Koplan B, Carmeli Y, Wright SB: Glycopeptides are no more effective than $\beta$-lactam agents for prevention of surgical site infection after cardiac surgery: a meta-analysis. Clin Infect Dis. 2004, 38:1357-1363. 10.1086/383318

31. Finkelstein R, Rabino G, Mashiah T, et al.: Vancomycin versus cefazolin prophylaxis for cardiac surgery in the setting of a high prevalence of methicillin-resistant staphylococcal infections. J Thorac Cardiovasc Surg. 2002, 123:326-332. 10.1067/mtc.2002.119698

32. Wood CA, Wisniewski RM: $\beta$-lactams versus glycopeptides in treatment of subcutaneous abscesses infected with Staphylococcus aureus. Antimicrob Agents Chemother. 1994, 38:1023-1026. 10.1128/aac.38.5.1023

33. Natsis NE, Cohen PR: Coagulase-negative Staphylococcus skin and soft tissue infections . Am J Clin Dermatol. 2018, 19:671-677. 10.1007/s40257-018-0362-9

34. Taha L, Stegger M, Söderquist B: Staphylococcus lugdunensis: antimicrobial susceptibility and optimal treatment options. Eur J Clin Microbiol Infect Dis. 2019, 38:1449-1455. 10.1007/s10096-019-03571-6

35. Sampathkumar P, Osmon DR, Cockerill FR 3rd: Prosthetic joint infection due to Staphylococcus lugdunensis. Mayo Clinic Proc. 2000, 75:511-512. 10.4065/75.5.511

36. Hou TY, Chiang-Ni C, Teng SH: Current status of MALDI-TOF mass spectrometry in clinical microbiology . J Food Drug Anal. 2019, 27:404-414. 10.1016/j.jfda.2019.01.001

37. Chiu KHY, Lam RPK, Chan E, Lau SKP, Woo PCY: Emergence of Staphylococcus lugdunensis as a cause of urinary tract infection: results of the routine use of MALDI-TOF MS. Microorganisms. 2020, 8:381. 
38. Kung BT, Seraj SM, Zadeh MZ, et al.: An update on the role of 18F-FDG-PET/CT in major infectious and inflammatory diseases. Am J Nucl Med Mol Imaging. 2019, 9:255-273.

39. Karambelkar P, Rojulpote C, Borja AJ, Youngs C, Bhattaru A: An unusual case of tricuspid valve infective endocarditis caused by Erysipelothrix rhusiopathiae. Cureus. 2020, 5:e7942. 10.7759/cureus.7942

40. Borja AJ, Hancin EC, Dreyfuss AD, et al.: 18F-FDG-PET/CT in the quantification of photon radiation therapy-induced vasculitis. Am J Nucl Med Mol Imaging. 2020, 10:66-73.

41. Rojulpote C, Seraj SM, Zadeh MZ, et al.: Role of FDG-PET/CT in assessing the correlation between blood pressure and myocardial metabolic uptake. Asia Ocean J Nucl Med Biol. 2020, 8:36-45.

42. Bhattaru A, Zhang V, H Emily, et al.: Potential applications of FDG-PET/MRI in detecting vascular dementia. J Nucl Med. 2020, 61:1149.

43. Bhattaru A, Borja A, Zhang V, et al.: FDG-PET/CT as the superior imaging modality for inflammatory bowel disease. J Nucl Med. 2020, 61:1159.

44. Kragsbjerg P, Bomfim-Loogna J, Törnqvist E, Söderquist B: Development of antimicrobial resistance in Staphylococcus lugdunensis during treatment-report of a case of bacterial arthritis, vertebral osteomyelitis and infective endocarditis. Clin Microbiol and Infect. 2000, 6:496-499. 10.1046/j.1469-0691.2000.00103.x

45. Merino P, Arribi A, Gestoso I, Picazo J, Gimeno L, Del Potro E: Linezolid treatment of a prosthetic joint infection with Staphylococcus lugdunensis in a patient with multiple myeloma. Int J Antimicrob Agents. 2010, 35:203-204. 10.1016/j.ijantimicag.2009.09.025

46. CLSI: Performance standards for antimicrobial susceptibility testing; twenty-third informational supplement. Clinical and Laboratory Standards Institute, Wayne, PA; 2013.

47. Anguera I, Del Río A, Miró JM, et al.: Staphylococcus lugdunensis infective endocarditis: description of 10 cases and analysis of native valve, prosthetic valve, and pacemaker lead endocarditis clinical profiles. Heart. 2005, 91:e10. 10.1136/hrt.2004.040659

48. Yeh C-F, Liu T-P, Cheng C-W, Chang S-C, Lee M-H, Lu J-J: Molecular characteristics of disease-causing and commensal Staphylococcus lugdunensis isolates from 2003 to 2013 at a tertiary hospital in Taiwan. PloS One. 2015, 10:e0134859. 10.1371/journal.pone.0134859

49. Ishiekwene C, Ghitan M, Kuhn-Basti M, Chapnick E, Lin YS: Staphylococcus lugdunensis endocarditis with destruction of the ventricular septum and multiple native valves. IDCases. 2017, 7:14-15. 10.1016/j.idcr.2016.10.011

50. Kyaw H, Raju F, Shaikh AZ, et al.: Staphylococcus Lugdunensis endocarditis and cerebrovascular accident: a systematic review of risk factors and clinical outcome. Cureus. 2018, 10:e2469. 10.7759/cureus.2469 